\title{
LUDO STRUYVEN
}

$\mathrm{KU}$ Leuven, Belgium

\section{Statistical profiling of unemployed jobseekers The increasing availability of big data allows for the profiling of unemployed jobseekers via statistical models}

Keywords: statistical profiling, long-term unemployment, benefit exhaustion, labor market discrimination

\section{ELEVATOR PITCH}

Statistical models can help public employment services to identify factors associated with long-term unemployment and to identify at-risk groups. Such profiling models will likely become more prominent as increasing availability of big data combined with new machine learning techniques improve their predictive power. However, to achieve the best results, a continuous dialogue between data analysts, policymakers, and case workers is key. Indeed, when developing and implementing such tools, normative decisions are required. Profiling practices can misclassify many individuals, and they can reinforce but also prevent existing patterns of discrimination.

\section{KEY FINDINGS}

\section{Pros}

๑ Systematic patterns between socioeconomic and sociodemographic variables, and the outcome of interest can be revealed by statistical models.

- Statistical models can direct future research on why some groups are more at risk, and on how the gap can be closed.

๑ Statistical profiling models offer an indication of the potential duration of an unemployment spell.

- Under some circumstances, statistical models can reduce existing patterns of discrimination.

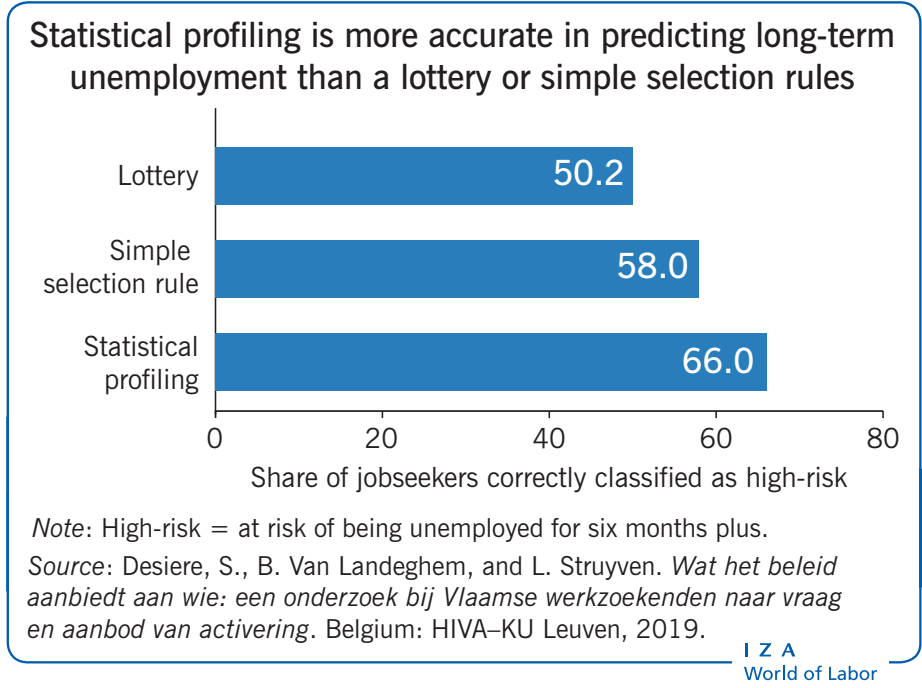

\section{Cons}

- The improvement in profiling accuracy when using statistical models as opposed to a lottery is modest and many individuals tend to be misclassified.

- Statistical profiling risks reinforcing existing patterns of discrimination.

- Current statistical profiling models predict outcomes, but do not reveal which program works for whom.

\section{AUTHOR'S MAIN MESSAGE}

Statistical profiling can help identify individuals at risk of becoming long-term unemployed and highlight appropriate predictive variables. However, such models do not unravel the mechanisms behind these relationships, and will hence not inform directly about suitable policies to tackle long-term unemployment. It is also not straightforward to evaluate whether targeted policies are effective. Additionally, policymakers who consider relying on statistical profiling to direct jobseekers to job counseling, training programs, or other social programs should evaluate the ethical implications: individuals are often misclassified and statistical profiling can reinforce patterns of discrimination. 


\section{MOTIVATION}

While the act of profiling jobseekers is as old as employment activation itself, the methods of profiling have changed profoundly.

Traditionally, employment services have profiled jobseekers in a rule-based manner, often segregating them into large general groups, such as younger versus older, and affording case workers some degree of discretion. More recently, however, governments are increasingly developing and implementing statistical profiling models based on administrative and/ or survey data to predict whether a jobseeker will become long-term unemployed. This development is in line with a broader expectation among governments to conduct evidencebased policy making, to prevent prolonged spells of joblessness, and to tailor services to individuals. Given the increasing popularity of statistical profiling and the increasing opportunities to build profiling models, it is useful to review these practices, to discuss how they might inform policy making, and to examine the potential moral implications policymakers face when implementing these models to target the unemployed.

\section{Key principles of statistical profiling: The statistical model and decision rules}

A statistical model returns an estimate of an individual's probability of exhausting benefits, the number of months spent in unemployment/on benefits, and so on. It is then up to researchers and policymakers to define decision rules or cut-off points that govern specific programs. For instance, assume a policymaker is interested in identifying new benefit claimants who are at risk of exhausting their benefits. Those for whom the predicted chance of exhausting benefits is larger than $50 \%$ could be categorized as highrisk, and those for whom the chance is lower than $50 \%$ as low-risk. But other cut-off values could also be used, such as $60 \%$ or $80 \%$. Alternatively, relative decision rules could be adopted which are dependent on the budget available to target a given set of individuals. The Kentucky Profiling model, for example, gives each individual a score from 1 to 20, where 1 refers to the $5 \%$ of individuals with the lowest predicted risk, and 20 refers to the $5 \%$ of individuals with the highest predicted risk (Black et al., 2007). Individuals with a score of 20 are invited to a mandatory training program, and then if the budget allows those with a score of 19 are invited, and so on until the budget is exhausted.

Source: Black, D. A., J. Galdo, and J. A. Smith. "Evaluating the Worker Profiling and Reemployment Services System using a regression discontinuity approach." American Economic Review 97:2 (2007): 104-107.

\section{Profiling versus targeting}

It is important to make a distinction between profiling and targeting. Statistical profiling aims to split the pool of jobseekers into homogeneous groups, groups of individuals who have the same chance of becoming long-term unemployed or to exhaust benefits. It is then up to stakeholders (the public employment service, policymakers, and so on) to decide how to use this information. One route is not to use it for operational purposes, but to look at the common characteristics that the model has identified for people who are more at risk than others. The model does not inform about why this is the case, nor how to effectively help these at-risk individuals. But at least this information might spark a debate, might direct future research, and eventually the development of policies that aim to support those in need. 
Another extreme is to actively use profiling to target individuals in a fully automated way. Those who are classified as high-risk are then directed to voluntary or mandatory active labor market programs, are set to be contacted more frequently by case workers, and so on. But again, the statistical profiling model will be able to identify at-risk individuals, but will not be able to show whether the interventions are effective. As a middle ground, the classification produced by the profiling practice can be used to inform case workers or the employment office, who can then use the classification as an additional source of information to make decisions regarding the coaching and monitoring they provide for an individual.

\section{Evaluating profiling practices}

Profiling, or a particular profiling practice, hinges upon the statistical profiling model and the decision rule that uses the predictions of the model as inputs to sort people into groups (e.g. low-risk and high-risk). A natural way to evaluate a profiling practice is to investigate how many people were correctly classified. Terms such as sensitivity (the percentage of those classified as high-risk who do eventually exhaust their benefits) and specificity (the percentage of those classified as low-risk who do not eventually exhaust their benefits) may be encountered. Obviously, sensitivity and specificity cannot be viewed in isolation, as there is a tradeoff between the two. If misclassification is less likely near the top and bottom of the ranking, making the high-risk pool smaller increases the sensitivity, but decreases the specificity and vice versa.

In general, it is preferable to observe that those with the highest profiling scores exhaust their benefits much more frequently than those with the lowest scores. A useful singleitem metric is the Profiling Score Effectiveness Metric (PSEM) (Sullivan, 2007), which can be calculated as follows:

$$
\text { PSEM }=1-(100-y) /(100-x)
$$

Where $x$ is the overall percentage of individuals who eventually exhaust their benefits, and $y$ the percentage of individuals with the highest profiling score who eventually exhaust their benefits.

If the model does not do any better than a lottery, the metric will be zero, whereas a perfect model will return a value of one. As an example, imagine a state where $20 \%$ of new benefit claimants will eventually exhaust their benefits. For the $20 \%$ of individuals with the highest profiling score (as predicted by a statistical model), $60 \%$ of individuals eventually exhaust their benefits. The PSEM for this model is then equal to:

$$
1-(100-60) /(100-20)=0.5
$$

Source: Sullivan, W. F., L. Coffey, L. Kolovich, C. W. McGlew, D. Sanford, and R. Sullivan. Worker Profiling and Reemployment Services Evaluation of State Worker Profiling Models: Final Report. ETA Occasional Paper No. 15, 2007.

\section{DISCUSSION OF PROS AND CONS}

\section{Transitioning from standard into advanced statistical profiling models}

Statistical profiling has traditionally been based on classic analytical techniques such as cross-tabulations or regression models [1]. However, novel machine learning techniques are increasingly being implemented in standard statistical software packages. This 
advancement in the field is facilitated by exponential improvements in computing power and rapidly growing availability of administrative data. These developments have offered public employment services such as the Flemish Employment and Vocational Training Office the opportunity to develop statistical profiling models using modern, data-hungry, and computationally expensive machine learning techniques [2]. These machine learning techniques are better at predicting which jobseekers are at risk of becoming long-term unemployed or of exhausting their benefits than standard regression models.

Academic research has certainly been an important source of inspiration for the development of statistical profiling. The academic literature in various disciplines across the social sciences has explored the determinants of unemployment duration or benefit exhaustion. These studies often provide very useful ideas for developing profiling models. However, building such a model remains a matter of trial and error and making ad hoc decisions. First, whether or not variables are good predictors will differ across countries. Second, in smaller-scale academic studies, variables (such as measures for soft skills) that are not always straightforward to collect at the population level (e.g. if this requires filling out extensive surveys) are often used. Finally, apart from practical hurdles, legal restrictions related to privacy protection can prevent policymakers from collecting, using, or merging data for the development of profiling models.

\section{Predictors of unemployment duration}

In 2000, a case study was conducted in Minnesota, USA, to investigate predictors of unemployment duration [3]. Although small-scale (989 usable observations), the study was rather influential as it inspired, for example, the Dutch Public Employment Service when it developed its profiling model. The case study recruited unemployment insurance claimants who were then re-interviewed one year later to track their labor market history. Variables found to be associated with lower reemployment success were being non-white, having worked with the previous employer for more than five years, and being female with children under the age of 18 . Variables associated with higher reemployment success were measures of economic need (number of children under the age of 18 and economic hardship). Other variables found to have explanatory power were a person's region of residence and occupation. The explanatory power of such macroeconomic variables has also been widely documented in policy reports discussing statistical profiling models based on administrative data. Interestingly, a large number of variables such as years of education, self-reported job search, and conscientiousness, were not found to be significant. This might be due to the small sample size, but there might also be several mechanisms at work that cancel each other out. For example, it is clear that a good labor market history, a resume with few gaps, and a short ongoing unemployment spell are very important for being successful in the labor market. But, as the results in this case study show, being employed with one employer for a long time predicts lower reemployment success, perhaps because skills have become obsolete or because these individuals are not used to negotiating with employers or being active in the job market. Having young children might create a need for higher income, or to accept a job more quickly and to increase search efforts, but as the above results suggest, opportunity costs can arise due to caring responsibilities. Finally, there can also be mediating effects, since variables are correlated with each other. For example, the authors found that conscientiousness predicts higher reemployment success once other variables are dropped from the model [3]. 
A similar project, but of a much larger scope, was set up by the Institute of Labor Economics (IZA) and is called the IZA Evaluation Dataset [4]. The IZA Evaluation Dataset is a German nationwide sample of 12 monthly cohorts of individuals who became unemployed between June 2007 and May 2008; the dataset tracks these individuals for 2.5 years after they entered unemployment. Regression analyses on these data show that higher school-aged educational attainment and further (professional) training are associated with fewer months spent in unemployment, as are being younger, having a strong employment record, having high numeracy skills, a high internal motivation or locus of control, being conscientious, being optimistic about finding a job, and having a good labor market history. Again, macroeconomic conditions (especially the local unemployment rate) can have strong predictive power.

It is interesting that in the literature, especially the economics literature, there are not many (influential) studies reporting exercises that are very close to statistical profiling of the unemployed. There are two main reasons for this. First, the international literature is often more interested in understanding how education and cognitive and non-cognitive skills are related to labor market success within the general population, and less so in examining a specific subsample of individuals who enter unemployment. Second, and more importantly, the economics literature is not just interested in models that accurately predict an outcome. The literature pays more attention to the mechanisms that actually lead to these outcomes. Take the Minnesota study above as an example, in which some variables appear to have an effect through different channels [3]. It is quite difficult to disentangle these effects; a setting is needed in which it is possible to evaluate what happens if one variable changes, all else being equal. Therefore, studies tend to focus on one factor at a time, rather than presenting a comprehensive model that accurately predicts ex post outcomes. But statistical profiling models offer a prediction of the unemployment spell for each individual, which, according to economic research, is very relevant information for jobseekers themselves. It is well-known that jobseekers are often too optimistic about their employment chances. These biased perceptions imply that they take different decisions with regard to their job search strategy than if they had the correct information [5].

\section{Normative implications of statistical profiling}

Before asking data analysts to build and implement a statistical profiling model, policymakers should define the outcome variable that the statistical model should predict (e.g. exhausting benefits, becoming long-term unemployed) and decide how they want to use this information. For example, policymakers could choose to route highrisk individuals to a mandatory training program, or to offer them subsidized training. However, this is not the endpoint of the policymaker's responsibility, as several key aspects must be considered when employing such models.

A first area of concern involves potential misclassifications produced by the model. In the last large-scale exercise to investigate profiling models used in different US states, the highest Profiling Score Effectiveness Metrics were around 0.25, and the lowest were below 0.1 [1]. As models will never perfectly predict an ex-post outcome, data analysts and policymakers must have a dialogue about which decision rule to implement, and how to trade sensitivity for specificity. Suppose a given government is very worried about 
people in need not getting the appropriate support while specific support is available for individuals who are categorized as high-risk. This government would hence be interested in profiling with a high specificity: those who are categorized as low-risk should indeed be those who are not exhausting their benefits ex post. If the statistical model provides predictions for the chance of exhausting benefits, the decision rule could then be adapted by choosing a lower cut-off value, above which individuals are categorized as high-risk.

Conversely, consider a post-election situation in which there is a regime switch: the new government wants to tighten budgets and might thus be worried about people having access to support who actually do not need it. This new government is hence interested in a profiling practice with a high sensitivity: those who are categorized as high-risk should indeed exhaust their benefits ex post. The new government could hence decide to increase the threshold value above which someone is classified as high-risk. The pool of high-risk individuals will then become smaller. If misclassification is less likely near the tails of the ranking, the sensitivity will increase. The specificity, however, will decrease, as the fraction of those who eventually exhaust their benefits will increase among the low-risk pool.

It is hence clear that different regimes might prefer different sensitivity-specificity combinations, and that the optimal combination (given the model) depends on how profiling is intended to be used. A mandatory program for the high-risk group might lead to a different conclusion than an opportunity for voluntary training for the high-risk group.

Another very important normative implication is the potential reinforcement of stigmatization of minorities. Statistical profiling models aim to predict outcomes but do not really focus on causal relationships. Good examples of this are the variables for someone's employment history. These often tend to be good predictors for unemployment duration, but as discussed above, do not say much about the mechanisms. When implementing these predictive models, however, it is still important to have an idea of the relationship between cause and consequence when it comes to understanding the normative implications of a profiling practice. A huge body of literature offers very convincing evidence that there is a causal relationship between race and the job finding rate, or, in other words, that labor market discrimination exists [6]. As a consequence, race can be an important determinant of whether or not someone exhausts their benefits. But is it therefore warranted to invite minority jobseekers more often to demanding mandatory training sessions? On the other hand, if profiling is primarily used to offer additional opportunities to vulnerable jobseekers (rather than to monitor job search), inviting proportionally more minority jobseekers may pose fewer ethical questions, and may even be considered positive discrimination [7].

If this relationship is causal, and ethnic minorities suffer from discrimination, policymakers might prefer not to reinforce such stigmatization. It is, however, not straightforward to avoid such reinforcement by statistical profiling models. In practice, public employment services complying with data privacy laws like the General Data Protection Regulation (GDPR) have adopted rules making it unlawful to include contentious variables such as gender, age, and ethnicity in the statistical model. However, these contentious variables are often correlated with other predictors, such as proxies for local labor market conditions. Indeed, minorities tend to be concentrated in certain areas. Recently, economists have proposed a simple methodology to partially mitigate the problem and have applied their suggestion to the Worker Profiling and Reemployment Services model in the US [8]. 
The main idea is that the contentious variables are still included in the model, but only at the prediction stage. The actual values of the latter variables are not used, but rather replaced with the average for the population. This practice avoids the possibility that other explanatory variables pick up the effect of labor market discrimination. Obviously, the predictive power of the model decreases, but the reinforcement of stigmatization does as well: in the study's empirical example, the percentage of black jobseekers in the high-risk group decreases from $22 \%$ to $16 \%$.

It is worth noting that after such adjustments, statistical profiling models might even prevent discrimination, especially when the alternative is granting case workers discretionary power to allocate people to programs. In the hiring context, for example, there is evidence that limiting hiring managers' discretionary power to overrule test results increases the quality of the pool of hired workers [9]. Algorithms are more transparent than the minds of decision-making humans, who often are prone to unconscious biases against certain groups [10].

\section{LIMITATIONS AND GAPS}

Profiling models reveal systematic patterns in unemployment duration, though the differences between people remain largely unexplained by the data. This means that such models do a bit better in classifying individuals ex ante than a lottery, but many individuals still tend to be misclassified ex post. Moreover, profiling models focus on predicting an outcome, and not on causal relationships. The latter implies that it can be difficult to interpret the results from a complex profiling model. Many variables in such models might also impact each other; for example, the level of education someone has will have had an impact on previously earned income. Including all these variables in a model will help policymakers to predict who is at risk of becoming long-term unemployed, but the model is not suitable to predict what will happen to unemployment duration if training vouchers are given to people with low levels of education, for example. While statistical profiling models might help identify at-risk people, they do not reveal which policy programs are effective for whom.

In any case, statistical profiling models will support rather than replace case workers. Many public employment services are currently experimenting with striking the right balance between automated and human-based decision making so that both approaches can reinforce each other. Finally, it is worth emphasizing that complex statistical profiling tools should ultimately improve the labor market outcomes of jobseekers. This does not yet appear to have been carefully evaluated in the academic literature, although studies have shown that statistical profiling rules can outperform case workers with regard to assigning jobseekers to the optimal program [11].

\section{SUMMARY AND POLICY ADVICE}

Matching survey data with administrative data can offer windows of opportunity to build more accurate statistical profiling models. While statistical profiling models are helpful to improve the identification of jobseekers who are at risk of becoming long-term unemployed, there are some caveats when it comes to their efficacy at better targeting unemployed individuals. Even if models contain a rich set of variables, a large number of individuals will be misclassified. Moreover, there is a risk of reinforcing the stigmatization 
of minority groups if used incorrectly. Policymakers need to maintain a consistent dialogue with researchers to determine the ideal trade-off between false positives and false negatives.

Statistical profiling can provide an additional source of information for case workers and public employment services. While it does not inform about causal relationships, it can help raise the question of why one group is more at risk than another. In this way, statistical profiling can serve as a guide to develop research projects that investigate causal mechanisms. Policymakers may want to promote the combination of statistical profiling with causal inference methods such as large-scale randomized controlled trials in combination with machine learning. For example, whether people at risk of becoming long-term unemployed really do benefit more from certain types of programs can be identified [12]. Or, with regard to (causal) machine learning techniques it might be investigated whether programs have heterogeneous treatment effects [11]. Instead of profiling jobseekers with respect to the predicted unemployment duration, they could then be profiled with respect to the predicted effectiveness of a program.

\section{Acknowledgments}

The authors thank two anonymous referees and the IZA World of Labor editors for many helpful suggestions on earlier drafts.

\section{Competing interests}

The IZA World of Labor project is committed to the IZA Code of Conduct. The authors declare to have observed the principles outlined in the code.

() Bert van Landeghem, Sam Desiere, and Ludo Struyven 


\section{REFERENCES}

\section{Further reading}

Arni, P., M. Caliendo, and R. Mahlstedt. Predicting the Risk of Long-Term Unemployment: What Can We Learn from Personality Traits, Beliefs and Other Behavioral Variables? Working Paper, 2014.

Loxha, A., and M. Morgandi. Profiling the Unemployed: A Review of OECD Experiences and Implications for Emerging Economies. World Bank Social Protection and Labor Discussion Paper No. SP1424, 2014.

\section{Key references}

[1] Sullivan, W. F., L. Coffey, L. Kolovich, C. W. McGlew, D. Sanford, and R. Sullivan. Worker Profiling and Reemployment Services Evaluation of State Worker Profiling Models: Final Report. ETA Occasional Paper No. 15, 2007.

[2] Desiere, S., K. Langenbucher, and L. Struyven. Statistical Profiling in Public Employment Services. OECD Working Paper No. 224, 2019.

[3] Wanberg, C. R., L. M. Hough, and Z. Song. "Predictive validity of a multidisciplinary model of reemployment success." Journal of Applied Psychology 87:6 (2002): 1100-1120.

[4] Arni, P., M. Caliendo, S. Künn, and K. F. Zimmermann. "The IZA Evaluation Dataset Survey: A scientific use file." IZA Journal of European Labor Studies 3 (2014): 1-20.

[5] Spinnewijn, J. "Unemployed but optimistic: Optimal insurance design with biased beliefs." Journal of the European Economic Association 13:1 (2015): 130-167.

[6] Bertrand, M., and S. Mullainathan. "Are Emily and Greg more employable than Lakisha and Jamal? A field experiment on labor market discrimination." American Economic Review 94:4 (2004): 991-1013.

[7] Desiere, S., and L. Struyven. "Using artificial intelligence to classify jobseekers: The accuracyequity trade-off." Journal of Social Policy (2020): 1-19.

[8] Pope, D. G., and J. R. Sydnor. "Implementing anti-discrimination policies in statistical profiling models." American Economic Journal: Economic Policy 3:3 (2011): 206-231.

[9] Hoffman, M., L. B. Kahn, and D. Li. "Discretion in hiring." Quarterly Journal of Economics 133:2 (2018): 765-800.

[10] Kleinberg, J., J. Ludwig, S. Mullainathan, and C. R. Sunstein. "Discrimination in the age of algorithms." Journal of Legal Analysis 10 (2018): 113-174.

[11] Cockx, B., M. Lechner, and J. Bollens. Priority to Unemployed Immigrants? A Causal Machine Learning Evaluation of Training in Belgium. IZA Discussion Papers No. 12875, 2019.

[12] Altmann, S., A. Falk, S. Jäger, and F. Zimmermann. "Learning about job search: A field experiment with job seekers in Germany." Journal of Public Economics 164 (2018): 33-49.

\section{Online extras}

The full reference list for this article is available from:

https://wol.iza.org/articles/statistical-profiling-of-unemployed-jobseekers

View the evidence map for this article:

https://wol.iza.org/articles/statistical-profiling-of-unemployed-jobseekers/map 